\title{
TWO INTEGRALS INVOLVING MODIFIED BESSEL FUNCTIONS OF THE SECOND KIND
}

\author{
by B. W. CONOLLY
}

(Received 20th January, 1955)

§ 1. Introductory. In $\S 2$ a product of two modified Bessel Functions of the Second Kind is expressed as an integral with a function of the same type as a factor of the integrand. In $\S 3$ an integral involving a product of these functions, regarded as functions of their orders, is evaluated in terms of another function of this kind. These results were suggested by a study of Mellin's inversion formula.

$\S 2$. Product of two modified Bessel Functions. The formula to be proved is

$$
\begin{aligned}
K_{m}(a) K_{n}(b) & \\
= & \int_{-\infty}^{\infty} e^{-u(m-n)}\left(\frac{a e^{u}+b e^{-u}}{a e^{-u}+b e^{u}}\right)^{\frac{1}{2}(m+n)} K_{m+n}\left[\sqrt{ }\left\{\left(a e^{u}+b e^{-u}\right)\left(a e^{-u}+b e^{u}\right)\right\}\right] d u,
\end{aligned}
$$

where $R(a) \geqslant 0, R(b) \geqslant 0, R(a+b)>0$.

This formula is a generalisation of a formula of Nicholson's (1), which can be deduced by putting $b=a$.

The proof is based on the formulae $(2,3)$

where $R(z)>0$, and

$$
K_{n}(z)=\frac{1}{2} \int_{-\infty}^{\infty} e^{-z \cosh t \pm n t} d t
$$

$$
K_{n}(z)=\frac{1}{2} z^{n} \int_{0}^{\infty} e^{-\frac{1}{\left(t\left(t+z^{2} / t\right)\right.} t^{-n-1}} d t
$$

where $R\left(z^{2}\right)>0$.

From (2), if $R(a)>0, R(b)>0$,

$$
K_{m}(a) K_{n}(b)=\frac{1}{4} \int_{-\infty}^{\infty} \int_{-\infty}^{\infty} e^{-a \cosh s-b \cosh t-m s+n t} d s d t
$$

Here make the transformation

and get

$$
s=u+v, t=u-v
$$

$$
\begin{aligned}
\frac{1}{2} \int_{-\infty}^{\infty} \int_{-\infty}^{\infty} e^{-a \cosh (u+v)-b \cosh (u-v)-u(m-n)-v(m+n)} d u d v \\
\quad=\frac{1}{2} \int_{-\infty}^{\infty} e^{-u(m-n)} d u \int_{-\infty}^{\infty} e^{-a \cosh (u+v)-b \cosh (u-v)-v(m+n)} d v .
\end{aligned}
$$

Now in the inner integral put $w=\left(a e^{u}+b e^{-u}\right) e^{v}$ and it becomes

where

$$
\left(a e^{u}+b e^{-u}\right)^{m+n} \int_{0}^{\infty} e^{-1\left(w+R^{2} / w\right)} w^{-m-n-1} d w,
$$

Thus, by (3), the inner integral is equal to

so giving formula (1).

$$
2\left\{\left(a e^{u}+b e^{-u}\right) / R\right\}^{m+n} K_{m+n}(R)
$$

§ 3. Integral of a Product of two modified Bessel Functions. The formula to be proved is 


$$
\begin{aligned}
& \frac{1}{2 \pi i} \int k^{-2 \zeta} \frac{K_{\mu+\zeta}(a)}{a^{\mu+\zeta}} \frac{K_{\nu-\zeta}(b)}{b^{\nu-\zeta}} d \zeta \\
& \quad=\frac{1}{2} k^{\mu-\nu}\left(\frac{k+k^{-1}}{a^{2} k+b^{2} k^{-1}}\right)^{t(\mu+\nu)} K_{\mu+\nu}\left[\sqrt{ }\left\{\left(k+k^{-1}\right)\left(a^{2} k+b^{2} k^{-1}\right)\right\}\right],
\end{aligned}
$$

where $a \neq 0, b \neq 0$ and the integral is taken up the entire length of the imaginary axis. The proof is based on the two following formulae $(4,6)$ :

$$
\left(\frac{1}{2} z\right)^{-n} K_{n}(z)=\frac{1}{8 \pi i} \int \Gamma\left(\frac{1}{2} \zeta\right) \Gamma\left(\frac{1}{2} \zeta-n\right)\left(\frac{1}{2} z\right)^{-\zeta} d \zeta,
$$

where the integral is taken up the imaginary axis with loops, if necessary, to ensure that the poles of the integrand lie to the left of the contour ; and

$$
\frac{1}{2 \pi i} \int \Gamma(a+\zeta) \Gamma(b-\zeta) z^{-\zeta} d \zeta=z^{a} \Gamma(a+b)(1+z)^{-a-b},
$$

where the integral is taken up the imaginary axis with loops, if necessary, to ensure that the poles of $\Gamma(a+\zeta)$ lie to the left and those of $\Gamma(b-\zeta)$ to the right of the contour. On replacing $\zeta$ by $\zeta-a$ the integral reduces to an $E$-function.

On substituting from (5) on the left of (4) and changing the order of integration it becomes

$$
\frac{2^{-\mu-\nu-4}}{(2 \pi i)^{3}} \int \Gamma\left(\frac{1}{2} s\right)\left(\frac{a}{2}\right)^{-s} d s \int \Gamma\left(\frac{1}{2} t\right)\left(\frac{b}{2}\right)^{-t} d t \int k^{-2 \zeta} \Gamma\left(\frac{1}{2} s-\mu-\zeta\right) \Gamma\left(\frac{1}{2} t-\nu+\zeta\right) d \zeta .
$$

From (6) it follows that the inmost integral is equal to

$$
2 \pi i k^{t-2 \nu} \Gamma\left(\frac{1}{2} s+\frac{1}{2} t-\mu-\nu\right)\left(1+k^{2}\right)^{-1 s-1 t+\mu+\nu} .
$$

Thus, on replacing $s$ and $t$ by $u+v$ and $u-v$ the expression reduces to

$$
\frac{2^{-\mu-\nu-3}}{(2 \pi i)^{2}} \frac{\left(1+k^{2}\right)^{\mu+\nu}}{k^{2 \nu}} \iint\left(\frac{a}{2}\right)^{-u-v}\left(\frac{b}{2}\right)^{-u+v} \Gamma\left(\frac{u+v}{2}\right) \Gamma\left(\frac{u-v}{2}\right) \Gamma(u-\mu-\nu)\left(1+k^{2}\right)^{-u} k^{u-v} d u d v .
$$

Now the last line may be written

$$
\int \Gamma(u-\mu-\nu)\left\{\frac{a b\left(1+k^{2}\right)}{4 k}\right\}^{-u} d u \int \Gamma\left(\frac{u+v}{2}\right) \Gamma\left(\frac{u-v}{2}\right)\left(\frac{a k}{b}\right)^{-v} d v
$$

and, from (6), on replacing $v$ by $2 v$, it is seen that the inner integral is equal to

$$
2 \pi i \times 2\left(\frac{a k}{b}\right)^{u} \Gamma(u)\left(1+\frac{a^{2} k^{2}}{b^{2}}\right)^{-u}
$$

Hence, on replacing $u$ by $\frac{1}{2} u$ and applying (5) the expression (A) becomes

where

$$
(2 \pi i)^{2} 4\left(\frac{1}{2} R\right)^{-\mu-\nu} K_{\mu+\nu}(R)
$$

and from this formula (4) follows.

$$
R=\sqrt{ }\left\{\left(k+k^{-1}\right)\left(a^{2} k+b^{2} k^{-1}\right)\right\}
$$

\section{REFERENCES}

(1) Nicholson, J. W., Quart. J. Math., 42 (1911), 220-223.

(2), (3) Watson, G. N., Bessel Functions, pp. 182, 183.

(4) Titchmarsh, E. C., Fourier Integrals, p. 197.

(5) MacRobert, T. M., Complex Variable, 4th ed., p. 374.

IJTORD, EssEX. 